\title{
Monte Carlo Simulations in Acousto-Photonic Imaging
}

\author{
Alex Nieva, Charles A. DiMarzio \\ Optical Science Laboratory - Northeastern University \\ 334 Egan Building \\ 360 Huntington Avenue \\ Boston, MA 02115 \\ 617-373-8570, 617-373-8970, cdimarzio@lynx.neu.edu \\ hitp://gnoson.ece.neu.edu/ osl \\ David Boas \\ Photon Migration Lab - Massachusetts General Hospital \\ Harvard Medical School 13th Street Building 149 \\ Charlestown, $M A$ 02129 \\ 617-724-0130,617-726-7422, dboos@nmr.mgh.harvard.edu \\ Ronald A. Roy, Sebastien Manneville \\ Boston University - Department of Aerospace and Mechanical Engineering \\ 110 Cummington Street, 1st floor \\ Boston, MA 02215 \\ 617-363-4846, ronroy@bu.edu
}

\begin{abstract}
Acousto-Photonic imaging (API) is a new technique for non-invasive medical imaging combining diffusive optical tomography (DOT) and focused ultrasound. Monte Carlo simulations are presented for the interaction of Near-Infrared light (NIR) and ultrasound in dense turbid media with high albedo.

(c) 2001 Optical Society of America

OCIS codes: (110.4500) Optical coherence tomography; (110.5120) Photoacoustic imaging; (110.7170) Ultra-
\end{abstract}

\section{Introduction}

Non-invasive optical imaging in human tissue is one of the most promising techniques to acquire infomation about tissue optical properties and its dynamics. Diffuse Optical Tomography (DOT) is a subject of active research, with multiple applications that range from oximetry and tissue spectroscopy to image of brain and breast tumors and brain function[1].

We have proposed a novel imaging technique that takes advantage of the properties of DOT and Ultrasound $[2,5]$. The main objective of the Acousto-Photonic imaging (API) is to use ultrasound to generate virtual diffuse optical sources to improve the spatial resolution as well as to acquire the optical properties of human tissue. The utility of this technique has been demonstrated by Wang et al.[3], Boccara et al.[4], DiMarzio et al.[5]. However, there is a lack of understanding of the real interaction of the diffuse waves and ultrasound. In the aim to understand how multiple scattered light and ultrasound interact we present a Monte Carlo model based on well developed algorithms for simulating light transport in tissue [6], but we include a first order approximation in the phase modulation due to the ultrasound. Results for simulations using $10^{6}$ photons in an infinite media using a point source are presented as well as future work using this technique.

\section{Monte Carlo approach}

A diffuse photon density wave is well explained by radiative transport theory [7], as well as numerical simulations based on the application of the Monte Carlo method [8], which consists of recording photon trajectories, while they are being scattered and absorbed in diffusive media. When we take into account the ultrasound we try to "tag" the photons whose paths take them deep into tissue. The number of photons interacting with the ultrasound is very small but the use of lock-in techniques for the detection help us to discriminate the tagged photons from the noise. In order to tag this photons we use a focused ultrasound beam modulated at $2.3 \mathrm{MHz}$. There are three basic models for the interaction mechanism in the API: modulation of the index of refraction due to the change of the particle density according to pressure changes of the ultrasound beam, periodic particle motion, and multiple speckle pattern modulation. 
In a Monte Carlo simulation process we see that the random walk of a particle is terminated if the particle is absorbed or if the particle leaves the region of interest. In order to simulate this behavior it is best to introduce statistical weights. In the simplest case, the weight is unity at the start of the simulation and since this is a diffuse photon density wave, it is decreased by the square root of the albedo, that is the ratio of the reduced scattering coefficient $\left(\mu_{s}^{\prime}\right)$ and the total interaction coefficient $\left(\mu_{t}=\mu_{s}^{\prime}+\mu_{a}\right)[9]$. In order to simulate the interaction between the sound and optical fields, we developed a first order approximation for the statistical weights of the carrier and various sidebands where the ultrasound is taken into account as a phase change in the diffuse waves generated at $70 \mathrm{MHz}$. We define the statistical weight for the API interaction as follows:

$$
W_{n i J}=\prod_{j=1}^{J}\left[A_{i j}+a_{i j}\right]\left[\exp \left(i k_{n} S_{i j}+i \vec{s}_{i j} \cdot \Delta \vec{k}_{n i j} e^{i \omega_{a} t}+i \vec{s}_{i j}^{*} \cdot \Delta \vec{k}_{n i j} e^{-i \omega_{a} t}\right)\right]
$$

where the capital letters represent static quantities due to the optical field, and the lower case letters represent variations at the acoustic frequency which in fact, are smaller than the optical quantities. $W_{\text {niJ }}$ is the statistical weight for one of the $n=9$ frequency domain sidebands that are generated due to the API signal. This is the value of the weight for the photon $i$ at frequency $n$ and scattering event $j$. The value of $\Delta k_{n i j}$ is defined as the vectorial diference between the wave vector of the incoming photon and its wave vector after the scattering event. The dot product of $\Delta k_{n i j}$ and the acoustic wave is responsible for the interaction between the ultrasound and the scattered light. For this preliminary model we are using a plane ultrasonic wave defined as $s_{i j}=s_{a} * \exp \left(i * \overrightarrow{k_{a}} \cdot \vec{r}\right)$. In future work we plan to use real simulations of the acoustic field for focused ultrasound. The phase change of the API signal is defined as $\Delta \phi_{n i j}=\vec{s}_{i j} \cdot \Delta \vec{k}_{n i j}$. Now if we do a first order approximation using Taylor series we have:

$$
W_{n i J} \approx \prod_{j=1}^{J}\left[A_{i j}+a_{i j}\right]\left[\exp \left(i k_{n} S_{i j}\right)\left(1+i s_{i j} \cdot \Delta k_{n i j} e^{i \omega_{a} t}\right)\left(1+i s_{i j}^{*} \cdot \Delta k_{n i j} e^{-i \omega_{a} t}\right)\right]
$$

Using the following mathematical calculations and approximations, we can express the weight as in equation 6 :

$$
\begin{aligned}
\prod_{j}\left[X_{j}+x_{j}\right]= & {\left[X_{1}+x_{1}\right]\left[X_{2}+x_{2}\right]\left[X_{3}+x_{3}\right] \ldots } \\
\approx & X_{1} X_{2} X_{3} \ldots+\left[X_{2} X_{3} \ldots\right] x_{1}\left[X_{1} X_{3} \ldots\right] x_{2} \ldots=\prod_{j}\left[X_{j}\right]\left[1+\sum_{j=1}^{J} x_{i j} / X_{i j}\right] \\
W_{n i J} \approx & {\left[\prod_{j=1}^{J} A_{i j}\right]\left[1+\sum_{j=1}^{J} a_{i j} / A_{i j}\right] \times\left[\prod_{j=1}^{J} \exp \left(i k_{n} S_{i j}\right)\right] } \\
W_{n i J} \approx & {\left[1+\sum_{j=1}^{J}\left(i s_{i j} / S_{i j} \cdot \Delta k_{n i j} e^{i \omega t}+i s_{i j}^{*} / S_{i j} \cdot \Delta k_{n i j} e^{-i \omega t}\right)\right] } \\
& {\left[1+\sum_{j=1}^{J}\left(i s_{i j} / S_{i j} \cdot \Delta k_{n i j} e^{i \omega t}+i s_{i j}^{*} / S_{i j} \cdot \Delta k_{n i j} e^{-i \omega t}\right)\right] }
\end{aligned}
$$



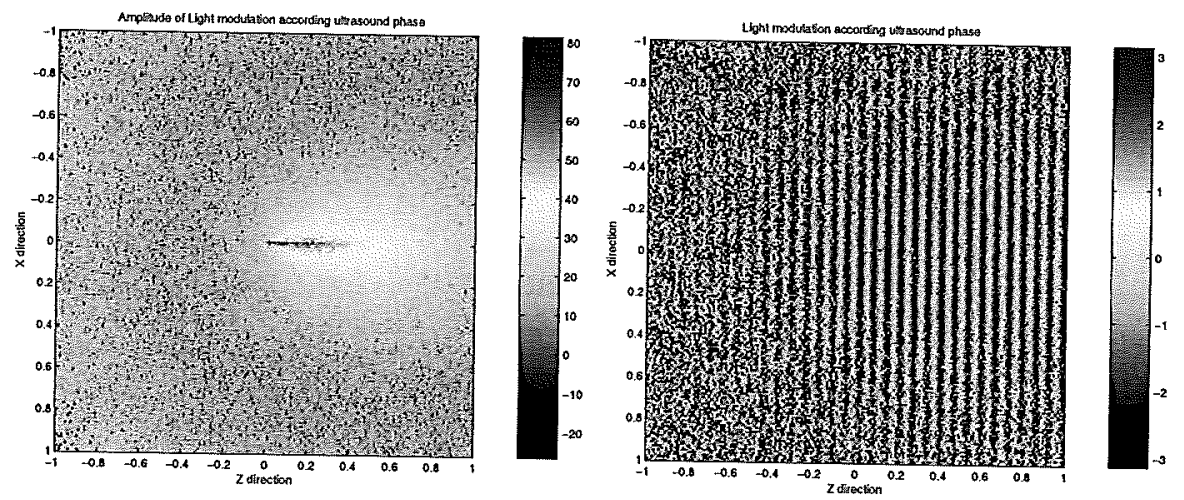

Fig. 1. Amplitude and phase modulation of diffuse light according to the behaviour of ultrasound in scattering media. We can see the behavior of the photons modulated by the ultrasound. The ultrasonic wavelength $(630 \mu \mathrm{m})$, is well defined and modulates the photons during scattering events.

\section{Simulation results}

In our experiments we modulate a laser light source at $72.3 \mathrm{MHz}$ (diode laser at $10 \mathrm{~mW}$ ), so that we can create a diffuse wave at this frequency. The laser light and the ultrasound beam are combined in a tank filled with titanium dioxide $\mathrm{TiO}_{2}$. In the Monte Carlo simulation we choose values for the optical properties of tissue that can resemble real ones. We consider $\mu_{a}=1 \mathrm{~cm}^{-1}$ and $\mu_{s}^{\prime}=10 \mathrm{~cm}^{-1}$ with an anisotropy factor $g=0.9$. The geometry for the Monte Carlo simulation is a cube of $2 \times 2 \times 2 \mathrm{~cm}$ and $100 \mu \mathrm{m}$ grid. We have used $10^{6}$ photons. We have a point light source located in the center of the sample volume irradiating in the $\mathrm{Z}$ direction, and the ultrasound axis is also in the $\mathrm{Z}$ direction.

Figure 1 presents the light modulation according to the phase of the ultrasound wave. This figure was plotted collapsing the volume in the $\mathrm{Y}$ direction doing the average of the non-zero valued voxels. We also present the amplitude of the diffuse wave.

\section{Conclusions and future work}

In this paper we have shown the mechanisms of interaction between ultrasound and diffuse light using a three dimensional Monte Carlo simulation of this kind of interaction. Current and future work is to take into consideration more realistic parameters, such as, focused ultrasound with different positions of the ultrasonic beam so that we can evaluate the SNR changes according to the spatial distribution of the API sources.

\section{References}

1. D.A. Boas, D.H. Brooks, E.L. Miller, C.A. DiMarzio, M. Kilmer, R.J. Gaudette, and Q. Zhang, "Imaging the body with diffuse optical tomography," in IEEE Signal Processing Magazine 18(6): 57-75 (2001).

2. 'T. J. Gaudette, "Acousto-photonic effect in scattering media," Master's Thesis, Northeastern University, (1998).

3. L. Wang, and X. Zhao, "Ultrasound-modulated optical tomography of absorbing objects buried in dense tissue-simulating turbid media," Appl. Opt. 36, 25 7277-7282 (1997).

4. S. Lévêque, A. C. Boccara, M. Lebec, and H. Saint-Jalmes, "Ultrasonic tagging of photon paths in scattering media: parallel speckle modulation processing" in Opt. Lett. 24, 3, 181-183 (1999).

5. C. A. Dimarzio, R. J. Gaudette, and T. J. Gaudette "New imaging technique combining diffusive photon density waves 6. D. A. Boas, ultrasound" in Optical Tomography and Spectroscopy of Tissue III Proc. SPIE 3597, 376-384 (1999).

6. D. A. Boas, M. A. 0'Leary, B. Chance, and A. G. Yodh, "Scattering of diffusive photon density waves bye spherical inhomogeneities within turbid media: Analytical solution and application," in Proc. Natl. Acad. Sci., USA 91, 4887-4891

7. Akira Ishimaru, Wave propagation and scattering in random media, IEEE Press, 1997. 8. S. A. Prahl, M. Keijzer, S. L. Jacques, and A. J. Welch, "A Monte Carlo model of light propagation in tissue," in SPIE
Institute Series, 5, 102-111 (1989).

9. I. V. Yaroslavsky, A. Yaroslavsky, H. Schwarzmaier, G. Akchurin, and V. Tuchin, "New approach to Monte Carlo simulation of photon transport in the frequency domain," in Proc. SPIE Photon Propagation in Tissues, 2626, 45-55 (1995). 